\title{
We Introduction Une dynamique de la vieillesse : vers le haut ou vers le bas
}

[ Simon-Daniel Kipman ${ }^{[1]}$

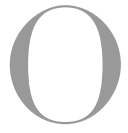

n ne peut plus considérer la vieillesse ni comme une crise, ni comme un état. C'est une période de la vie, que maintenant on tend à scinder encore et encore : troisième, quatrième, cinquième âge..., grand âge, jeune retraité et personne âgée dépendante.

Nous n'entrerons pas dans ce genre de discussion, dont il ne reste d'ailleurs que des miettes de vieillesse. Retenons seulement qu'il s'agit d'une période et que, tout en restant un moment de vie, elle répond à ses propres lois évolutives, à ses propres critères, à ses propres contraintes... quand bien même celles ci seraient inspirées des lois de tout un chacun. Nous sommes tous égaux devant les lois, humaines, républicaines, ou de nature... à condition que ces lois soient adaptées et tiennent compte de la situation.

Si l'on se réfère au beau titre du colloque, la vieillesse n'est pas que déchéance pour ou par rapport à l'adulte, elle est, elle peut être dynamisante pour le vieux, et pour ceux qui s'en approchent, à condition de s'entendre sur le sens de cette dynamisation. Tout est question de conditionnement ; c'est le paquet qui fait le cadeau ; c'est la sauce qui fait passer le poisson. Interrogeons notre présentation des choses. Est-ce que j'écris ici comme vieux (et justifiant mon intervention), comme psychiatre (la vieillesse n'est pas une maladie mentale), comme psychanalyste, tenant compte de son " contre transfert".

Quatre pistes sont possibles :

A/ La vieillesse n'est pas la mort. Même si la mort est au bout du chemin (de tous les chemins), il convient de ne pas anticiper le deuil. Sinon c'est un deuil pathologique.

[1] Président de l'Observatoire Francophone de la Médecine de la Personne s.d.kipman@wanadoo.fr

Derniers titre parus: L'oubli et ses vertus, Albin Michel 2013; Dictionnaire critique des termes de psychiatrie et de santé mentale, Doin.

Article disponible sur le site http://www.psycho-clinıque.org ou nttp://ax.dol.org/10.1051/psyc/201540007 
Quand on entend des spécialistes du grand âge, on est frappé de ce que leurs discours se terminent presque systématiquement par la mort, surtout s'ils ne sont pas vieux eux même. Cela signifie-t-il que les personnes âgées nient, dénient la proximité de leur propre fin? Ou bien que les adultes projettent leurs peurs sur les vieux qu'ils rencontrent. Personne n'aime envisager la disparition de ses parents. Personne ne peut éviter de voir leur image dans les plus vieux qu'il rencontre. Personne n'aime envisager ses parents amoindris : qui pourrait alors encore se protéger. "Allo, maman, bobo ", n'a pas d'âge. Alors qui se trompe? Qui trompe qui ?

Le fait (j’insiste : le fait) que chacun, face à un vieux, pense à sa mort (je laisse là l'ambiguïté propre à la langue française), enferme le vieux dans cette pensée, dans cette perspective... et donc le pousse vers la mort (l'immobilité, la dépendance). On, nous faisons, en torturant nos théories, une sorte de deuil anticipé, qui facilite l'abandon ressenti par beaucoup de personnes âgées en situation d'isolement. C'est probablement là une des racines de ce que l'on appelle la dépression des personnes âgées, comme un élément quasi constant du vieillissement.

B/ La vieillesse n'est pas une maladie, mais pourtant elle requiert des médecins et surtout des soignants. (Voir mon texte dans le numéro de polémique sur la vieillesse. Doin, 2010). Il n'est pas discutable que l'aire d'activité, l'aire de jeu des personnes âgées est diminuée par rapport à leurs performances d'adultes et de jeunes adultes ; il est certain qu'ils sont atteints de maladies, troubles sans doute plus facilement que d'autres, mais on ne peut s'appuyer, pour affirmer cela, ni sur l'indice de mortalité (voir supra), ni sur ce constat éminemment étonnant que la dernière année de la vie coute plus cher, en termes de santé, que toutes les autres. Ce serait plutôt à comprendre comme une interrogation sur d'éventuels acharnements thérapeutiques, sur la confusion entretenue par la surprotection pathogène entre assistance sociale et médecine. On ne parle jamais, en tous cas en Europe, des étonnantes aptitudes nouvelles des personnes âgées.

Ce déficit (par rapport aux critères “ importés » des aptitudes adultes) de capacités, d'aptitudes à se défendre des agressions internes et externes exige-t-il des soins particuliers?

- oui s'il s'agit de prendre en compte une maladie déclarée, en tenant compte des impératifs de la période de vie. Par exemple, s'il faut mobiliser très vite un opéré, cette mobilisation ne peut être aussi forcée qu'à d'autres moments de la vie.

- s'il s'agit de prévention de maladies susceptibles de survenir, il convient de tenir compte de la qualité de vie et de l'espérance de vie. Les préventions primaires sont souvent à très long terme. Les dépistages systématiques seront nuancés en fonction là encore de l'espérance de vie. Bref, une véritable prévention ne peut s'appuyer que sur une clinique individualisée et revisitée pour cette tranche d'âge.

C/ La vieillesse est celle des survivants, des durs, qui ont surmonté bien des choses. Le troisième et le quatrième âge sont les âges des cicatrices et des souvenirs anciens. 
Et, comme on le sait ces souvenirs ne sont pas tous roses, bien au contraire (voir mon livre L'oubli, à paraître).

La moyenne d'âge des personnes entrant dans les établissements pour personnes âgées, dépendantes ou non, est de plus en plus élevée. Nous mourrons de plus en plus vieux, sans jamais pourtant atteindre l'éternité et l'immortalité. Mais il n'empêche que ceux qui en arrivent là, dans cette dépendance aggravée par l' “ internement " ressenti à leur entrée en EHPAD, et par les soins constants et sans commune mesure avec ce qu'ils vivaient auparavant (ce qui ne peut que leur donner une piètre idée d'eux même) sont des survivants. Des gens qui ont traversé de multiples épreuves de la vie, la dernière étant l'arrachement à leurs habitudes.

La vieillesse est une période de découvertes (voir blog du 9 avril 2012). Les vieux ont du temps et de la disponibilité : les laisse-t-on toujours en profiter?

Tous les retraités le disent: ils ont du temps libre ou libéré, mais ils ne peuvent réaliser (enfin ou en fin) leurs rêves anciens. Ils n'en sont plus capables, mais ils ont à leur disposition des sources d'information qui peuvent les lancer, si on les autorise ou s'ils s'y autorisent, à se lancer sur des pistes nouvelles. Je me souviens de mon grand père, qui après avoir regardé la télévision, me demandait de lui expliquer les règles du football (ce que j'étais bien incapable de faire !).

Mais leur expérience et leur curiosité sont disponibles.

“Si jeunesse savait, si vieillesse pouvait » le dicton peut s'entendre autrement: jeunesse a besoin de la “ sagesse » des vieux pour agir (c'est ainsi que l'on considère les personnes âgées dans d'autres formes de société, dans lesquelles le gain immédiat n'est pas au premier plan, des “ oncles » vietnamiens aux gérontocrates chinois). Du coup la vieillesse devient l'âge de la liberté de penser, de la liberté de découvertes (j’ai oui dire que les personnes âgées qui en ont les moyens voyageaient beaucoup). La vieillesse est l'âge de l'intellect, si la jeunesse est celui de l'action.

La psychanalyse là dedans, a toute sa place :

\footnotetext{
"Le professeur Freud est passé refermez donc vos brises bises Rien de fait sans la psychanalyse. Vous direz ce que vous voudrez Pour un progrès c'est un progrès ” Aragon, Le roman inachevé.
}

Modification du transfert. On connaît l'histoire du transfert, d'abord considéré comme un artéfact, il est devenu un des moteurs essentiels de la cure et, sous un mode élargi, de toute la théorisation psychanalytique, associé en cela au contre transfert. Mais pour des raisons de simplification évidente, mais aussi de projection personnelle de ceux qui en usent, on a pris la mauvaise habitude d'en parler comme d'un “ objet en soi » : transfert positif, négatif, paternel, maternel, etc. en oubliant 
de le corréler à la situation du moment pour dire vite. Or la situation du moment pour une personne âgée est assez particulière pour induire des formes de transfert assez précises.

Les modalités du transfert des personnes âgées sont marquées par au moins deux paramètres :

- le besoin d'aide, la dépendance sous toutes ses formes, qui implique un appel contre transférentiel, une soif de transfert positif et infantile chez tous ceux qui se sentent ou à qui l'on dit qu'ils sont “ diminués ";

- un besoin d'autonomie, un repli narcissique du marginalisé.

Ce double mouvement transférentiel des personnes âgées sur ceux qui s'en occupent (qu'ils soient aidants naturels ou soignants, que ce soit dans le cadre d'une psychothérapie réglée ou dans n'importe quelle relation d'aide (qui a toujours sa dimension psychothérapeutique) crée un vacillement dans la demande et dans la réponse des personnes âgées aux propositions d'aide et de soin qui leur sont faites. Il serait utile de prendre en compte ce vacillement qui met a mal le contre transfert spontané des soignants qui est un des éléments d'une meilleure compréhension de ce qui se passe souvent si mal en institution spécialisée.

Modification du contre transfert. On ne peut s'empêcher de projeter sur les personnes âgées que l'on rencontre un certain nombre d'associations toutes personnelles et individuelles, et des associations collectives quasi obligatoires. Ce que j’appelle les fantasmes forcés.

Quand il s'agit de personnes âgées, personne n'admet sereinement qu'ils soient des images parentales plus ou moins déchues. Il faudrait qu'ils continuent à être vaillants pour qu'on puisse soit s'appuyer sur eux, soit s'y opposer. Mais quand ils deviennent dépendants, cela devient dangereux pour nous : bientôt notre tour? Edipe face à son père?

Le contre transfert du soignant s'en trouve profondément modifié, et alourdi des idées toutes faites sur cette situation.

Méthodes adaptées. On ne le répétera jamais assez : la psychanalyse est à la fois une théorie générale du psychisme, une méthode psychothérapique standardisée (la cure type). Cette méthode, et ses adaptations fidèles (les psychothérapies dites " réglées ») ne répondent pas facilement aux besoins exprimés par les personnes âgées :

- les cure type sont “ hors temps » alors que le temps dévolu aux personnes âgées est limité ;

- elles ne se fondent que sur une relation langagière laissant la dimension corporelle à distance : or les personnes âgées sont sans cesse persécutées par leurs limitations corporelles, leur douleurs et souffrances. Ce sont des hypochondiaques obligés ;

- les personnes âgées ne sont pas toujours aussi mobiles, ni aussi riches que ces méthodes l'exigent : c'est avec des personnes âgées et invalidées (provisoirement ou définitivement) que j’ai pu expérimenter les psychothérapies à domicile ; 
- perte de mémoire et immédiateté, répétition. On a beaucoup glosé sur les pertes de mémoire des personnes âgées, sur leurs troubles du langage. Je m’en expliquerai davantage dans un travail en cours sur l'oubli.

Mais je fais aussi référence aux expériences menées par Florence Quartier à Genève (Un avenir pour la vieillesse, Doin, éd.) sur la relation thérapeutique qui peut s'installer entre des “ déments séniles » et des soignants apparemment mal préparés.

D'ailleurs, on se rend compte que dans la vulgate psychanalytique, dans laquelle les expériences infantiles, précoces (ou souvent couvertes par ce que l'on appelle l'amnésie infantile) sont plus immédiatement accessibles aux personnes âgées. C'est d'ailleurs peut-être, un des paramètres, de ce que l'on appelle leur labilité émotionnelle, car ces expériences infantiles sont très chargées, surchargées d'affects.

Bref, ce pour quoi je plaide, c'est pour une véritable refondation de la clinique psychiatrique, médicale des personnes âgées (un peu comme le travail qui a été fait, en psychiatrie, par les psychiatres d'enfants il y a quelques décennies), en liaison avec les efforts de classification moderne (voir La médecine de la personne, Doin Arnette, qui vient de paraître). Je plaide pour une clinique respectueuse de la personne concernée, ce qui implique un travail sur soi même, sur l'observateur et le classificateur.

C'est la chance de la gériatrie que d'être à ce point la proie de fantasmes collectifs qu'elle puisse, par retour devenir un exemple ou un modèle pour l'ensemble de la médecine. 